\title{
LA RECEPCIÓN DE ROUSSEAU EN LA FORMACIÓN INICIAL DEL MAGISTERIO PRIMARIO (ESPAÑA, SIGLO 20)
}

\author{
Antonio Viñao Frago \\ Universidad de Murcia, España.
}

\section{$\operatorname{Cos} 80$}

\section{A RECEPÇÃO DE ROUSSEAU NA FORMAÇÃO INICIAL DO MAGISTÉRIO PRIMÁRIO (ESPANHA, SÉCULO 20)}

\section{Resumo}

Neste artigo é abordada a imagem e as ideias de e sobre Rousseau transmitidas, na Espanha, ao magistério primário durante o século 20. A análise é feita a partir de manuais de História da Educação e de outras fontes similares. Após analisar as fontes utilizadas e seus destinatários, se expõe a imagem e a idéia de Rousseau como pai da pedagogia moderna, predominante nos escritos pedagógicos da época para, em seguida, examinar o lugar e o tratamento dado à Rousseau. Esse exame é feito a partir da periodização que leva em conta duas épocas: a anterior à guerra civil e a posterior, com a finalidade de mostrar o predomínio, sobre tudo durante o franquismo, de uma visão católico-conservadora, moralizante e crítica de Rousseau.

Palavras-chave: Rousseau, manuais escolares, educação primária, formação do magistério primário, Espanha século 20.

\section{RECEPCION OF ROUSSEAU IN FORMATION INITIAL OF PRIMARY SCHOOL TEACHERS (SPAIN, $20^{\text {th }}$ century)}

\begin{abstract}
This article deals with Rousseau's images and ideas present in the school books of History of Education, and other similar sources, used in the training of primary school teachers in Spain during the $20^{\text {th }}$ century. After analyzing the sources used and their addresses, the predominance of Rousseau's ideas and image as the father of modern pedagogy in the pedagogical literature of this period is explained. Immediately after, by way of contrast, Rousseau's place and the way of considering him in the above mentioned school books are examined. The above analysis is divided into two periods, before and after the civil war, in order to show the predominance of the catholicconservative moralizing and critical approach, particularly during francoism.
\end{abstract}

Key-words: Rousseau, school books, primary education, teacher's training, Spain $20^{\text {th }}$ century. 


\section{LA RECEPCIÓN DE ROUSSEAU EN LA FORMACIÓN INICIAL DEL MAGISTERIO PRIMARIO (ESPAÑA, SIGLO 20)}

Resumen

Este artículo versa sobre la imagen e ideas de y sobre Rousseau transmitidas en España al magisterio primario durante el siglo 20, a través de los manuales de Historia de la Educación y otras fuentes similares. Tras analizar las fuentes utilizadas y sus destinatarios, se expone la imagen e idea de Rousseau como padre de la pedagogía moderna, predominante en los escritos pedagógicos de la época, para seguidamente, y a modo de contraste, examinar el lugar y el tratamiento dados a Rousseau en los mencionados manuales. Dicho examen se divide en dos épocas: la anterior a la guerra civil y la posterior, con el fin de mostrar el predominio, sobre todo durante el franquismo, de una visión católico-conservadora, moralizante y crítica de Rousseau.

Palabras-clave: Rousseau, manuales escolares, educación primaria, formación del magisterio primario, España siglo 20.

\section{LA RECEPCION DU ROUSSEAU ET LA FORMATION INITIALE DES ENSEIGNANTES PRIMAIRES (ESPAGNE, siècle $20^{\mathrm{eme}}$ )}

\section{Résume}

Cet article porte sur les images et les idées de et sur Rousseau transmises aux instituteurs en Espagne au cours du 20ème siécle, à travers des manuels d'Histoire de l'Education et d'autres sources similaires. Après avoir analisée les sources utilisées et ses destinataires, on expose l'image et idée de Rousseu comme père de la pédagogie moderne predominant dans la littérature pedagogique de cette époque. Ensuite, on examine le lieu et le traitement donné à Rousseau dans les manuels susnommés. Cette étude est divisée dans deux étapes: l'antérieure et la postérieure a la guerre civile, afin de montré la prédominance, surtout pendant le franquisme, de l'approche catholique-conservatrice, moralisant et critique, sur le genevois.

Mots-clé: Rousseau, manuels scolaires, enseignement primaire, formation des instituteurs, Espagne $20^{\text {ème }}$ siècle. 
a mayoría de los estudios sobre la recepción de Rousseau en España versan sobre la difusión e influencia de sus escritos e ideas políticas o filosóficas y de su producción literaria. No existe un análisis específico de dicha difusión e influencia en el campo de la educación, aunque información y observaciones sobre el particular se hallen presentes en estudios de índole general como el ya clásico libro de J. R. Spell sobre Rousseau en el mundo hispánico antes de $1833^{1}$ o en otros más concretos como, por ejemplo, en cualquier trabajo sobre Pedro Montengón o el Eusebio, un tema en el que parece inexcusable referirse a las influencias en el autor o en dicha obra del Emilio. Este texto trata un aspecto específico de esa difusión e influencia educativo-pedagógica: la imagen e ideas de y sobre Rousseau transmitidas en España al magisterio primario en la disciplina de Historia de la Educación desde comienzos del siglo 20, que es cuando esta materia se incluye en los planes de estudio de las escuelas normales, hasta 1991 año en que desaparece al diluirse en la de Teorías e Instituciones Contemporáneas de la Educación.

Su alcance cuantitativo y cualitativo no es baladí. Los aproximadamente 60.000 maestros y maestras que en 1936, al comienzo de la guerra civil, ejercían como tales, o los más o menos 260.000 que regentaban una escuela a comienzos de la década de los 90 del pasado siglo, habían cursado una asignatura llamada Historia de la Pedagogía desde 1901 a 1950 de modo independiente, y desde 1971 a 1991 como una parte de la disciplina de Pedagogía -, Educación y su Historia - desde 1950 a 1967 - o Historia de la Educación - desde 1967 a 1971 -, en la que con mayor o menor extensión, con un tratamiento u otro, la figura de Rousseau formaba parte de los autores de necesaria inclusión en su código o canon disciplinar. Constituía, en suma, una referencia obligada, asimismo presente en los cuestionarios oficiales de la prueba final para la obtención de los títulos de maestro o maestra, cuando existieron, o en las contestaciones a los temarios de oposiciones de acceso al magisterio primario.

Desde un punto de vista cualitativo, además, la imagen obtenida de Rousseau en su formación inicial en las escuelas normales, combinada o no con otras lecturas, se integraba en el imaginario profesional, en la visión que, como maestros, tenían de su actividad y de la pedagogía como ciencia y como arte o práctica. Rousseau era un referente a seguir e imitar, a tener en cuenta o a rechazar y criticar. Si, como se decía en 1918 en uno de los manuales de la asignatura, el ginebrino era "la figura más importante del siglo 18 y ocupa un lugar indiscutible en la historia de la pedagogía" (Puigserver, 1918, p. 77), la imagen e ideas sobre Rousseau que el magisterio primario tuviera en mente, cobran una especial relevancia.

En un texto de este tipo no cabe un análisis exhaustivo del tema. Lo limitaremos a dos cuestiones: el encaje o encuadre de Rousseau en relación con las ideas, autores y acontecimientos de su época o inmediatamente anteriores y posteriores, y el tratamiento general dado a su vida y obra. Dos aspectos que, según se reconocerá en muchos de los manuales de la disciplina, están indisolublemente relacionados. Antes de efectuar dicho análisis es necesario, sin embargo, realizar algunas precisiones sobre las fuentes

\footnotetext{
${ }^{1}$ SPELL, Jefferson Rea. Rousseau in the spanish world before 1833. New York: Gordian Press, 1938. Reimpreso en 1969 por Gordian Press. Hist. Educ. [Online] 
utilizadas, sus características, sus posibilidades y sus límites, así como sobre los destinatarios de las mismas.

\section{Las fuentes utilizadas y sus destinatarios}

Para la realización de este trabajo se han consultado, aunque no se mencionen en las notas a pie de página, un total de 39 manuales de la disciplina fechados entre 1903 y 1984, 4 programas, 8 libros de contestaciones a los temarios de oposiciones al magisterio primario y 1 a la prueba final o reválida, y 4 cuestionarios oficiales de la materia. Dado que, de hecho, los programas solo ofrecen el índice a seguir en su estudio, y que las características de las contestaciones y cuestionarios les asemejan, en este punto, a los libros de texto, las consideraciones que se hacen sobre el uso de estos últimos como fuente son extensibles al resto de la documentación utilizada.

Los libros de texto no son ensayos o estudios monográficos. Antes al contrario, ofrecen una versión simplificada, muy estructurada, de lo que se considera esencial o básico en una disciplina en función de la edad y tipo de alumnado. Todo ello, además, debe encajar en un temario que tenga un cierto orden o coherencia. Lo habitual, una vez que el código de la disciplina se configura, es que abunden las repeticiones, los lugares comunes, por no decir los plagios y copias, entre unos y otros manuales, así como que un mismo manual conozca un número elevado de ediciones sin experimentar cambios sustanciales $^{2}$, que alguno de ellos se constituya en modelo a seguir por los restantes, o que más de uno sea el resultado de las notas y apuntes de clase tras varios años de enseñanza.

El esquema seguido en la práctica totalidad de los manuales en relación con los autores relevantes, suele seguir un cierto orden: vida y obras, influencias recibidas, exposición del contenido de sus obras más destacadas, influencias ejercidas y valoración crítica - errores y aciertos, validez actual de sus propuestas. Todo ello, en relación con un solo autor, puede alcanzar, en unos casos, las treinta y tantas páginas y, en otros, verse reducido a una sola página o varias líneas de ella.

La presencia de la Historia de la Educación o de la Pedagogía en los estudios de magisterio tenía, a diferencia de otras materias, una finalidad más formativa que profesional en un sentido estricto. Con independencia de ello, hay que tener en cuenta la edad y formación de aquellos a quienes iba dirigida. Desde 1901 a 1931 la disciplina era cursada por estudiantes de 17 o 18 años, que por lo general, tras la enseñanza primaria, habían superado un examen de ingreso.

El plan de 1931, al exigir el título de bachiller para cursar los estudios de Magisterio, elevó tanto el nivel cultural del alumnado como su edad en unos dos o tres años. De nuevo, tras la guerra civil, el plan de 1942 permitió la realización del examen de ingreso en las Escuelas Normales o del Magisterio con 12 años cumplidos, con lo que la Historia de la Pedagogía podía ser cursada, en último año de la carrera, por estudiantes de 16 o 17 años con una débil e insuficiente formación académica. La elevación de la edad mínima de los 12 a los 14 años en 1945 no mejoró mucho las cosas.

\footnotetext{
${ }^{2}$ Tal sería el caso, por ejemplo, de la muy influyente Historia de la educación y de la pedagogía del jesuita Ramón Ruiz Amado, en la que lo escrito sobre Rousseau en la primera edición (Barcelona, Gustavo Gili, 1911 , p. 218-253) coincide con lo que figura en la $12^{\mathrm{a}}$ edición publicada cincuenta años más tarde, hacia 1960.

\begin{tabular}{|c|c|c|c|c|c|}
\hline Hist. Educ. [Online] & Porto Alegre & v. 17 & n. 41 & Set./dez. 2013 & p. 11-28 \\
\hline
\end{tabular}
}


Habría que esperar a 1967 para que volviera a exigirse el título de bachiller para el ingreso en las escuelas del magisterio, y a la ley general de educación de 1970 para añadir a dicho título el Curso de Orientación Universitaria y la superación de la prueba de selectividad si se quería acceder a las entonces llamadas Escuelas Universitarias de Formación del Profesorado de Educación General Básica. Estas diferencias en la edad y en la formación del alumnado, junto con otras de índole ideológica, explican la menor calidad formativa, con alguna excepción, de los manuales de la disciplina publicados en los años 40 y 50 del siglo pasado, así como de las contestaciones a los temarios de oposiciones de dichos años.

\section{Otras fuentes de información: Rousseau en la bibliografía y revistas pedagógicas del primer tercio del siglo 20}

Rousseau no era, por supuesto, un desconocido en el mundo de la pedagogía y de la enseñanza primaria cuando la Historia de la Pedagogía se incluye, como materia formativa, en los planes de estudio del magisterio primario a comienzos del siglo 20 . Es cierto que a partir del primer tercio del siglo 19, cuando en los años 30 y 40 de dicho siglo se configura el sistema educativo español tal y como ha perdurado, con algunos cambios, hasta el último tercio del siglo 20, Rousseau no constituye un punto de referencia para quienes tenían que crear y dar forma al sistema educativo del nuevo régimen liberal. El Rousseau del Emilio difícilmente podía servir de ejemplo si de lo que se trataba era de crear un sistema educativo desde el Estado, y los escritos rousseaunianos que podían ser de utilidad en este sentido, el Rousseau defensor de la educación pública nacional, estatal de las Consideraciones sobre el gobierno de Polonia o el Discurso sobre la economía política, no habían sido traducidos al español y eran prácticamente desconocidos ${ }^{3}$. No obstante, es posible hallar, durante el siglo 19, referencias críticas, neutras o laudatorias a Rousseau en los manuales de la disciplina de Pedagogía que los maestros y maestras cursaban en las escuelas normales ${ }^{4}$.

El que puede considerarse padre fundador de esta disciplina en España, Pablo Montesino, menciona solo dos veces al ginebrino en el curso de educación que impartió en la Escuela Normal de Maestros a partir de 1839 para incluirle, junto a Quintiliano, Milton y Locke, entre los autores de los "pocos buenos tratados de educación" conocidos por entonces en España, o para calificarlo como "un célebre escritor sobre educación" (Montesino, 1988, p. 82 y 130) pero la influencia de sus ideas y de su obra, a través sobre todo de Pestalozzi y Fellenberg y en relación con su filantropismo y la importancia que concedía a la educación física, es ampliamente reconocida ${ }^{5}$.

De un modo u otro, en la España de mediados del siglo 19 y por lo que a la educación se refiere, Rousseau había devenido ya un clásico a leer, mencionar o citar, pero no era un autor a tener en cuenta en lo que atañe a la configuración del sistema educativo, ni en relación con la organización escolar y los sistemas y métodos de

\footnotetext{
${ }^{3}$ Las Consideraciones sobre el gobierno de Polonia no serían traducidas y editadas en España hasta 1985 y el Discurso sobre la economía política hasta 1989.

${ }^{4}$ Por ejemplo, RABAZAS ROMERO, Teresa. Los manuales de pedagogía y la formación del profesorado en las escuelas normales de España (1839-1901). Madrid: Uned, 2001, p. 220, 240, 260 y 275, entre otras.

${ }^{5}$ Por ejemplo, SUREDA GARCÍA, Bernat. Introducción. En: MONTESINO, Pablo. Liberalismo y educación del pueblo. Madrid: Biblioteca Nueva, 2006, p. 13-52.

Hist. Educ [Online]

\begin{tabular}{l|l|l|l|} 
v. 17 & n. 41 & Set./dez. 2013 & p. $11-28$
\end{tabular}
}


enseñanza a implantar o seguir. Como afirmaba en 1844 Laureano Figuerola sobre el Emilio en su Guía lejislativa e inspectiva de instrucción primaria, una obra útil, según constaba en su portada, "para autoridades, comisiones locales y provinciales, inspectores y maestros de instrucción primaria",

Este romance sobre la educación, esta utopía de un hombre de genio, de un escritor de mediocre instrucción y poca experiencia, pero de elocuencia irresistible, es más bien un libro teórico que práctico. El Emilio, bueno para hacer reflexionar sobre la educación, es inútil para servir de guía al visitador [inspector] de escuelas populares. (Figuerola, 1844, p. 170)

La sentencia estaba dictada: Rousseau era un autor cuya mención mostraba la cultura pedagógica de quien a él se refería. Era, por así decir, una referencia obligada en cualquier tratado pedagógico. Su lectura y conocimiento resultaba inexcusable para quien quisiera o tuviera que teorizar sobre la educación, pero no era nada útil en relación con la práctica escolar, con la organización del aula y, en definitiva, con esa nueva ciencia - arte del magisterio primario, de índole teórico-práctica, que era la pedagogía.

Quien, en todo caso, tomará a Rousseau en el último tercio del siglo 19 como punto de partida de una orientación o línea pedagógica seguida por Pestalozzi, Froebel y Spencer, entre otros, de la que se consideraban continuadores, será la Institución Libre de Enseñanza. Así lo indicaba Juan Uña en el discurso de inauguración del curso 1882 1883 en el colegio de la institución ${ }^{6}$, y así lo afirmarían Francisco Giner de los Ríos - que en el Congreso Nacional Pedagógico de 1882 calificaría a Rousseau ante un amplio auditorio de pedagogos y maestros, de "padre de la pedagogía moderna" (Congreso Nacional Pedagógico, 1883, p. 188) y Manuel Bartolomé Cossío en diversas ocasiones y escritos, sobre todo en relación con la importancia de la educación física, los trabajos manuales o las actividades al aire libre y en la naturaleza ${ }^{7}$.

Nada tiene por ello de extraño que otro destacado institucionista, Luis de Zulueta, realizara en 1910 su tesis doctoral sobre "La pedagogía de Rousseau y las percepciones del espacio y el tiempo", o que incluyera el Emilio en 1921 entre los "doce mejores libros" (Zulueta, 2006, p. 67) de una biblioteca de obras fundamentales en la historia de la humanidad, en respuesta a una cuestión planteada por el Ateneo Enciclópedico Popular de Barcelona en el curso 1915 a $1916^{8}$. O que otro relevante institucionista, Domingo Barnés, ministro de Instrucción Pública durante la Segunda República, escribiera hacia 1920 sobre el ginebrino estas palabras:

La labor entera de Rousseau cae dentro de la Pedagogía. Si para un político su obra esencial sería El contrato social y para un literato La nueva Eloísa, el Emilio sería la más importante, no sólo para el pedagogo, sino

\footnotetext{
${ }^{6}$ UÑA, Juan. Discurso leído por el Ilmo. Sr. D. Juan Uña, rector de la Institución Libre de Enseñanza, en la inauguración del actual curso académico. Boletín de la Institución Libre de Enseñanza (Bile), n. 136, 1882, p. 221-223.

7 GINER DE LOS RÍOS, Francisco. Obras completas. XVI. Ensayos menores sobre educación y enseñanza. Tomo I. Madrid: Espasa-Calpe, 1927, p. 270, y Obras completas. XII. Educación y enseñanza, Madrid: Espasa-Calpe, 1933, p. 105, 179, y 198 (textos de 1884, 1885 y 1886), y COSSÍO, Manuel B. De su jornada: fragmentos. Madrid: Imprenta de Blass, 1929, p. 177-180.

8 DE ZULUETA, Luis. El ideal en la educación: ensayos pedagógicos y otros textos. Madrid: Biblioteca Nueva, 2006, p. 67 (1ª edición de 1921). Edición y estudio introductorio de Victoria Robles Sanjuán.

\begin{tabular}{|c|c|c|c|c|c|}
\hline Hist. Educ. [Online] & Porto Alegre & v. 17 & n. 41 & Set./dez. 2013 & p. 11-28 \\
\hline
\end{tabular}
}


para aquel que hiciera la historia de la cultura general. Además, en las tres obras aparece el educador: el educador del pueblo en El contrato social; el educador de la vida - espontánea, sentimental - en La nueva Eloísa, y el educador del individuo en el Emilio, cuyos principios pedagógicos son aplicables a las demás obras. (Barnés, s. a., p. 129)

Los manuales de Historia de la Pedagogía, cuando esta disciplina nace, no eran, además, los únicos textos en los que el magisterio primario español podía hallar información sobre el Rousseau pedagogo. Otros textos y escritos contribuyeron a configurar el imaginario rousseauniano del magisterio, e influyeron asimismo en lo que del ginebrino se decía en dichos libros de texto. Por de pronto, cuando se publicaron los primeros manuales españoles de Historia de la Pedagogía, los de García Barbarín en 1901 y Miguel Farga, quizás en $1903^{9}$, se disponía ya de la edición en castellano de la Historia universal de la pedagogía de Paroz, aparecida en 1887, en cuya tercera edición, de 1889, el editor, en una nota inicial dirigida "al magisterio", decía que "nada prueba tanto la corriente afición del Magisterio Español a toda suerte de estudios pedagógicos, y la importancia de la presente obra, como la rapidez con la que se han agotado la primera y la segunda edición" (Paroz, 1889, p. 130) y de la Historia de la pedagogía de Hailman publicada en 1894, a las que seguirían años después las historias de la educación de Davidson (1910), Painter (1911), Damseaux (1911), Guex (1912), Weimer (1914), Monroe (1918 - 1919) y Compayré (1920). Es decir, toda una serie de libros, de distinta factura y orientación, en los que las siempre amplias referencias al ginebrino serían citadas, copiadas o parafraseadas en buena parte por los manuales españoles de la época y por más de uno de los posteriores ${ }^{10}$.

Además, el Emilio estaba accesible al lector en castellano. A las ediciones ya existentes - las más cercanas en el tiempo eran las de Madrid de 1862 y 1879 -, se sumarían en estos años las de Paris (1896 y 1916, Garnier Hermanos, traducción de Ricardo Viñas), 1916 (Madrid, Daniel Jorro, traducción de Rafael Urbano), hacia 1925 (Barcelona, Maucci, traducción de José Marchena) y, ya durante la II República, las de 1932 (Madrid, Librería Bergua, traducción de José Marchena) y 1933 (Ávila, Tipografía de Senén Martín, traducción de José Marchena), además de la Antología de Rousseau preparada por Ma Luisa Navarro, editada por la Revista de Pedagogía en 1931 en su colección de La pedagogía clásica ${ }^{11}$.

No creemos, sin embargo, que la lectura del Emilio fuera la vía de acceso habitual entre el magisterio español al pensamiento e ideas pedagógicas de Rousseau. Incluso puede plantearse, como hipótesis, que Rousseau fuera, entre quienes se preocupaban

\footnotetext{
${ }^{9}$ GARCía BARBARíN, Eugenio. Historia de la pedagogía con un resumen de la española. Madrid, 1901, y MIGUEL FARGA, Luis. Compendio de historia de la pedagogía. Barcelona, s.a., pero hacia 1903.

${ }^{10} \mathrm{La}$ adscripción, por ejemplo, de Rousseau a la corriente o tendencia de la educación humana abstracta o general adoptada por N. Escanilla en su Guía para el estudio de la pedagogía histórica (Salamanca, 1933, p. 241), y por los cuestionarios oficiales de la materia aprobados por O. M. de 8 de septiembre de 1933, parece estar tomada de la Historia de la pedagogía de Franklin Verzelius Newton Painter (Madrid: Daniel Jorro, 1911, p. 314), y las referencias al probable suicidio de Rousseau en algunos de los manuales de tendencia católico-conservadora del franquismo, de GUEX, François. Historia de la instrucción y de la educación. Madrid: 1912, p. 181.

${ }^{11}$ A comienzos del siglo 20 los escritos de Rousseau traducidos al español se reducían, además del Emilio, a El contrato social, Pigmalión, el Discurso sobre el origen y los fundamentos de la desigualdad entre los hombres, Julia o La Nueva Eloísa, los Pensamientos y las Confesiones.
}

\begin{tabular}{|c|c|c|c|c|c|}
\hline Hist. Educ. [Online] & Porto Alegre & v. 17 & n. 41 & Set./dez. 2013 & p. 11-28 \\
\hline
\end{tabular}


por las cuestiones educativas, un autor más citado o mencionado que leído. Y conocido, sobre todo a partir de los años 20 , por ser uno de los padres de la pedagogía moderna y del movimiento internacional de la Escuela Nueva, según se repetía en manuales y libros. Razón por la cual su nombre había sido dado, y quedaría ligado en el futuro y en la mente del magisterio primario, al Instituto pedagógico, creado en 1912, ubicado en Ginebra y considerado el organismo científico más representativo de dicho movimiento. Al Instituto Jean-Jacques Rousseau que, en los años 1920 y 1930, se convertiría en lugar de peregrinación obligada, en forma de visitas y estancias, por cuantos pedagogos, profesores y maestros deseaban conocer los últimos avances y tendencias en el campo de la enseñanza.

Así, en efecto, junto a los libros de Compayré y Vial sobre el Rousseau pedagogo ${ }^{12}$, traducidos y publicados en España en aquellos años, y la ya mencionada y poco difundida tesis doctoral de Luis de Zulueta, los artículos aparecidos en las revistas pedagógicas más prestigiosas de la época - escasos en comparación con los dedicados, por ejemplo, a Pestalozzi que, a diferencia de Rousseau, sí era un docente - proceden de algunas de las figuras más relevantes, en España y fuera de ella, del movimiento de la Escuela Nueva o del campo científico de la paidología ${ }^{13}$, o se refieren al Instituto Jean-Jacques Rousseau de Ginebra.

La revista La Escuela Moderna, que en sus páginas dedicó bastantes trabajos a las figuras más destacadas de la historia de la pedagogía, en sus 45 años de existencia, entre 1891 y 1934, sólo publicó dos artículos sobre Rousseau, por lo demás poco relevantes, frente a cuatro, aparecidos entre 1920 y 1924, sobre los hombres, las ideas y las actividades del Instituto ginebrino que llevaba su nombre. No es de extrañar que en la mente del magisterio primario de la época, al menos entre los más innovadores de dicho colectivo, Rousseau fuera visto, sobre todo, como el precursor del movimiento de la Escuela Nueva, la paidología y el paidocentrismo. Que constituyera, como se ha dicho, "un punto de partida, nunca un punto final". Que su "presencia" en la pedagogía y prácticas innovadoras del magisterio primario en el primer tercio del siglo 20 - una presencia combinada con la de autores como Spencer y Dewey, entre otros, fuera más "un horizonte - un estado del espíritu o el espíritu de la época - que una realidad coherente y sistemática" (Torrano; Pujadas, 2012, p. 151).

Un ambiente o espíritu pedagógico innovador, el de estos años, que, entre otros aspectos que también remitían al ginebrino, enfatizaba la vida al aire libre, el rechazo de los libros de texto, el activismo, el recurso a la observación y experiencia directa y, sobre todo, el paidocentrismo. Paradójicamente, esta imagen, la de padre fundador o punto de partida de la pedagogía moderna, no será la que predomine, ni siempre se corresponde con la que ofrecían los manuales de Historia de la Pedagogía anteriores a la guerra civil y, sobre todo, los inmediatamente posteriores. Esta paradoja o contraste requiere ser explicado.

\footnotetext{
${ }^{12}$ COMPAYRÉ, Gabriel. J. J. Rousseau y la educación natural. León: La Nueva Editorial, s. a. pero 1907, y VIAL, F. La doctrina educativa de J. J. Rousseau. Barcelona: Labor, 1931.

${ }^{13}$ CLAPARĖDE, Edouard. Las ideas pedagógicas de Rousseau y el concepto funcional de la infancia, Bile, n. 646, 1914, p. 1-8, J. J. Rousseau y al concepción funcional de la infancia, Bile, n. 766, 1924, p. 7-14, y n. 767, 1924, p. 42-50, y Rousseau y la significación de la Infancia, Revista de Pedagogía, n. 1, 1922, p. 121-131; D. Barnés: Rousseau como precursor de la paidología, Bile, n. 683, 1917, p. 35-38.

\begin{tabular}{l|l|l|l|l|l|} 
Hist. Educ. [Online] & Porto Alegre & v. 17 & n. 41 & Set./dez. 2013 & p. 11-28
\end{tabular}
}




\section{Rousseau: de enciclopedista revolucionario y filantropista a naturalista}

¿En qué corrientes o tendencias filosóficas y/o pedagógicas se incluye a Rousseau en los manuales de Historia de la Pedagogía? ¿Cómo se le etiqueta o califica? ¿Con qué otros autores o educadores se le relaciona? ¿A quiénes precede o antecede?

Con independencia de aquellas adscripciones genéricas a la pedagogía del siglo 18 que nada nos dicen, en los manuales del primer tercio del siglo 20 Rousseau es situado unas veces entre los enciclopedistas y/o revolucionarios, junto a La Chalotais, Condorcet, Condillac, Helvetius, Diderot y Voltaire ${ }^{14}$; otras veces, entre los filantropistas, junto a Basedow, Campe y otros, a quienes precede ${ }^{15}$. En ocasiones es un precursor de la revolución francesa; en otras, el origen del filantropismo. En otras, es a la vez ambas cosas: aparece con o detrás de los enciclopedistas y revolucionarios y antes del filantropismo, de Basedow, de Pestalozzi y de Kant ${ }^{16}$. En otros casos, es situado junto y tras Locke, precediendo a Kant y Basedow como continuadores del ginebrino, (Puigserver, 1918) o en una línea filosófica calificada genéricamente de moderna, tras Bacon, Descartes y Locke y antes de Kant, Fichte y Herbart (De Tudela, 1919). Por último, en uno de las manuales con mayor número de ediciones, el de Gil Muñiz y Pertusa Peris, se le dedica al ginebrino un amplio y único capítulo al inicio del estudio de la pedagogía en el siglo 18 al que, ya desde el comienzo, se le califica como el siglo de Rousseau ${ }^{17}$.

Tras la guerra civil y hasta los años 70 del siglo pasado es posible observar ciertas continuidades con el período precedente. Así, por ejemplo, hay quien sitúa a Rousseau entre los precursores de la revolución francesa, junto a los enciclopedistas o inmediatamente antes o después de ellos ${ }^{18}$. Otros autores lo asocian a la corriente filantrópica o filantrópico - naturalista, precediendo a Basedow y, en ocasiones, a Pestalozzi. ${ }^{19} \mathrm{Su}$ inclusión en un mismo tema del cuestionario de oposiciones, en 1944, junto a Pestalozzi y Herbart propició este tipo de conexión, claramente forzada en el caso de Herbart. ${ }^{20}$

${ }^{14}$ Por ejemplo, en GARCÍA BARBARín, Eugenio. Historia de la pedagogía con un resumen de la española. Madrid, $2^{a}$ edición, 1907, p. 169-178, ESCRIBANO HERNÁNDEZ, Godofredo. Historia de la pedagogía. Madrid, 1910, p. 87-93, RECUERO GARCÍA, Gutiérrez. Historia de la pedagogía. Valencia, s. a., pero hacia 1920, t. I, p. 115-121, y ABENZA, A. Programa de historia de la pedagogía. Barcelona, 1932, p. 5.

${ }^{15}$ DíAZ MUÑOZ, Pedro. Historia de la pedagogía. Valladolid, $2^{\mathrm{a}}$ edición, 1919, p. 115-119, RUIZ AMADO, Ramon. Historia de la educación y de la pedagogía. Barcelona, Gustavo Gili, 1911, p. 288-305, y SANTA MARÍA Y SÁENZ, Maria C. Historia de la pedagogía y de la educación. Badajoz, 1930, p. 171-180. En este último caso, tanto en 1930 como en la $2^{2}$ edición de 1941, Tolstoy es el autor que sigue a Rousseau dentro de la corriente filantrópica, incluso antes de referirse a los filantropistas alemanes de finales del siglo 18 y comienzos del 19.

${ }^{16}$ Por ejemplo, en MIGUEL FARGA, Luis. Compendio de historia de la pedagogía, op, cit., p. 416-440, y en los cuestionarios oficiales de la materia aprobados por O. M. de 8 de septiembre de 1933.

${ }^{17}$ GIL MÚNIIZ, Antonio y PERTUSA PERIS, Vicente. Pedagogía moderna. Tomo III. Historia de la educación y de la pedagogía, Córdoba, 5a edición, 1930, p. 331-349.

18 SOLANA, Ezequiel. Curso completo de pedagogía: cuarta parte. historia de la pedagogía. Madrid: Escuela Española, 1944, p. 257-274.

${ }^{19}$ ALONSO FERNÁNDEZ, L. Estudios elementales de pedagogía: tomo segundo. Historia de la pedagogía. Madrid, 1946, p. 153-162, Edelvives: Historia de la pedagogía. Zaragoza: Luis Vives, 1965, p. 27-42; LLOPIS, J. Historia de la educación. Barcelona, 1969, p. 173-185, y GUTIÉRREZ ZULUAGA, Isabel. Historia de la educación. Madrid: Narcea, 3 ed., 1970, p. 293-305.

20 Contestaciones al cuestionario oficial de oposiciones al Magisterio Nacional. Madrid: El Magisterio Español, s. a. pero 1944, t. I, p. 728-735, y Contestaciones al cuestionario oficial de las oposiciones al Magisterio Nacional. Madrid: Escuela Española, 1944, t. III, p. 508-523.

\begin{tabular}{|c|c|c|c|c|c|}
\hline Hist. Educ. [Online] & Porto Alegre & v. 17 & n. 41 & Set./dez. 2013 & p. 11-28 \\
\hline
\end{tabular}


En otros casos, los autores se iban más allá en el tiempo y establecían una larga línea de conexión desde Bacon al filantropismo pasando nada más y nada menos que por Ratke, Comenio, Hobbes, Locke, Berkeley, Hume, Bayle, Montesquieu, Voltaire, los enciclopedistas y Rousseau o, más directamente, desde Bacon, Comenio y Locke a Rousseau y Pestalozzi ${ }^{21}$. Sin embargo, lo más peculiar de esta nueva generación de manuales es la caracterización de Rousseau, en casi todos ellos, como el origen o el máximo o único representante del naturalismo pedagógico. Una corriente u orientación que según algunos autores, continuarían y desarrollarían Pestalozzi, Froebel y sobre todo Spencer ${ }^{22}$.

\section{Identificación exclusiva y restrictiva del Rousseau pedagogo con el autor del Emilio}

Para los manuales de Historia de la Pedagogía Rousseau es, por lo general, el autor del Emilio y poco más, por no decir nada más. Son mayoría los autores que reducen su análisis o exposición de su obra pedagógica al Emilio, como lo son aquellos que no relacionan dicha obra con otras del ginebrino o, esta vez menos, los que no mencionan ninguna otra, ignorando lo que sostenía Domingo Barnés, hacia 1920, al afirmar que para entender el Emilio había "que estudiar el resto de sus obras, y aún su vida, especialmente en sus Confesiones, en Rousseau, juez de Juan Jacobo y en los Ensueños de un paseante solitario" (Barnés, s. a., p. 128).

Cuando el Emilio se relaciona con otros escritos es bien para establecer una breve comparación con El contrato social, y afirmar que el primero representa en el mundo de la educación lo que el último en el de la política; bien para destacar que el período en que Rousseau vivió en L'Ermitage, sería "el más fecundo de su vida" ya que en él escribiría sus tres obras más relevantes, Julia o La Nueva Eloísa, El contrato social y el Emilio ${ }^{23}$, o incluso para establecer una cierta conexión ideológica entre ellas ${ }^{24}$; o bien, por último, para derivar del concepto de naturaleza expresado en el Discurso sobre el origen y los fundamentos de la desigualdad entre los hombres, las directrices tanto de El contrato social como del Emilio.

Otras menciones a los dos discursos de Dijon, las Confesiones o las Cartas desde la montaña o la ópera El adivino de la aldea, son más escasas y puntuales. Sólo en el caso

${ }^{21}$ ABELLÁN, D. Pedagogía: educación y su historia. Murcia, 1954, p. 289-301, y MONTILLA, F. La educación y su historia. Madrid, 1952, p. 198-203.

${ }^{22}$ La lista sería muy extensa. Además de los manuales de Llopis, Zuluaga, Abellán y Montilla mencionados en las notas 28 y 30, habría que incluir, entre otros, los de MÁLAGA GARCÍA, E. Historia de la pedagogía. Burgos: Hijos de Santiago Rodríguez, s. a., pero hacia 1947, p. 141-152, VEGA ALONSO, Francisco. Educación y su historia, s. I., s. a. pero hacia 1952, p. 157-160, SANDÍN, Celestino. Historia de la educación. Alcoy: Marfil, 1967, p. 78-83. Sobre la clara adscripción de Rousseau al naturalismo pedagógico bajo el franquismo, y después de él, basta con decir que no solo está presente en los manuales de mayor difusión y número de ediciones (por ejemplo, en los de F. Montilla de 1957 con $7^{\text {a }}$ edición en 1964, Gil Muñiz de 1960 con 11ª edición en 1966, I. Gutiérrez Zuluaga de 1968 con 4ª edición de 1972, y Moreno, Poblador y del Río de 1971 con 4a edición en 1986), sino también, y esto es tanto o más relevante, en los cuestionarios oficiales de la reválida de Magisterio de los años 50 y de oposiciones al magisterio o a escuelas de localidades de más de 10.000 habitantes de los años 40 y 50 del siglo pasado. Oficialmente Rousseau no solo pasa a ser un naturalista, sino también el naturalista por excelencia o, en todo caso, el padre del naturalismo pedagógico.

${ }^{23}$ FLORIANO CUMBREÑO, Antonio C. Los problemas educativos ante la historia. Historia de la pedagogía. Madrid: Magisterio Español, s a., pero hacia 1943, t. II, p. 168-169.

${ }^{24}$ Por ejemplo, en DÍAZ MUÑOZ, Pedro. Historia de la pedagogía, op. cit., p. 117. 
de Floriano Cumbreño, ya hacia 1943, se alude a las Consideraciones sobre el gobierno de Polonia y al Discurso sobre la economía política con su defensa de "la Educación concebida como una función del Estado" (Floriano Cumbreño, 1943, p. 172), a modo de contrapunto a lo dicho en el Emilio; y en otro caso, el del manual de la editorial Luis Vives, publicado hacia 1960, se alude al Proyecto para la educación de M. de Sainte-Marie con el fin de desprestigiar la labor de Rousseau como preceptor ${ }^{25}$.

En síntesis, Rousseau es, ante todo y sobre todo, el autor del Emilio; esta será la obra cuyo contenido se exponga libro a libro, con mayor o menor detalle, en los manuales de Historia de la Pedagogía o Educación; y esta obra, junto con su vida, será aquella por la que será valorado, juzgado, criticado o ensalzado. Pero esta cuestión, la del tratamiento dado a Rousseau en dichos manuales, y la imagen que ofrecen del ginebrino, será tratada en el siguiente epígrafe.

\section{Tratamiento e imagen de Rousseau}

En el análisis del tratamiento dado a Rousseau en los libros de texto de Historia de la Pedagogía o de la Educación y de la imagen que ofrecen del mismo, distinguiré dos períodos: el anterior a la guerra civil (1901-1936) y el posterior. Ello no implica cortes o rupturas significativas. Más bien, lo que se aprecian son continuidades y, en todo caso, aspectos que permiten efectuar algunas observaciones clarificadoras.

1901-1936: entre la visión católico-conservadora y la renovadora

Rousseau no deja indiferente a nadie. Como decían Gil Muñiz y Pertusa y Périz, en uno de los manuales más ecuánimes de estos años,

para tratarlo pedagógicamente se han publicado infinidad de trabajos de mérito, pero la mayor parte de las veces con un sentido apasionado; ya convirtiéndose en apologistas intransigentes que han justificado los mayores errores y vicios de este filósofo, ya con sentido de enemistad implacable, censurándolo todo, o, al menos, no concediéndole importancia, ni aún a sus ideas más geniales, algunas de las cuales han trascendido de modo indiscutible a nuestra sociedad. (1930, p. 331)

Entre quienes consideraban que el Emilio era "una antorcha" con la que "los que se hallaban desorientados ven y se orientan", cuya "memoria sería imperecedera", y quienes solo veían "en esta obra un conjunto de quimeras y utopías dignas de ser quemadas", hay quienes pretenden "examinar" su obra de modo "lo más imparcialmente [...] posible" (Escribano Hernández, 1925, p. 243). Difícil cometido. Si en los primeros manuales pueden hallarse juicios de distinto signo - desde quienes le reconocen "grandes errores" junto a "hermosas ideas" (Miguel Farga, 1903, p. 419.) hasta quienes recuerdan que "contiene impiedades y errores opuestos a la doctrina católica", entre ellos el de negar el pecado original, por lo que su obra no podía ser puesta "en manos de quienes no tuvieran arraigadas sus convicciones religiosas" (Edelvives, 1960, p. 40), la Historia de la Educación y de la Pedagogía del jesuita Ruiz Amado vino a sentar en 1911 las bases de la visión católico-conservadora del Rousseau pedagogo. Unas bases repetidas en manuales posteriores, y ancladas en la afirmación, primero, de un probable suicidio y, después, en un párrafo que no precisa comentario alguno:

${ }^{25}$ EDELVIVES. Historia de la pedagogía. Barcelona: Luis Vives, s. a., pero hacia 1960, t. II, p. 40. \begin{tabular}{l|l|l|l|l|l}
\hline Hist. Educ. [Online] & Porto Alegre & v. 17 & n. 41 & Set./dez. 2013 & p. 11-28
\end{tabular} 
Este hombre, inconstante en todas sus empresas, inútil para el oficio de educador, inmoral, embustero, ladrón, que llevó a sus hijos a la inclusa, ha venido a ser, por una extraña burla de la suerte, jel padre de la educación y Pedagogía modernas. (Ruiz Amado, 1911, p. 291) ${ }^{26}$

Una vez enviado Rousseau al infierno, ya era posible caracterizarlo como "hombre singular, digno de lástima" (García Barbarín, 1907, p. 173) o "el hombre de las contradicciones" (Ibid., 178) de "desarreglada conducta", con evidentes "pruebas de locura" y "libertino", (Díaz Muñoz, 1919, p. 119.) "la antítesis personificada entre sus acciones y sus escritos" (García Barbarín, 1907, p. 173), causante de la muerte de su madre, sentimentalista bucólico, o escritor poco original, sino plagiario, de Montaigne, Fenelon y Locke, entre otros autores (Ruiz Amado, 1911).

En otras ocasiones, tras un tímido reconocimiento general se añadían unas palabras descalificadoras. Así, el Emilio venía a ser, en el mejor de los casos, "una amalgama de errores y verdades" (Casas y Sánchez, 1910, p. 98) en la que los "grandes errores" y "enormes disparates" (Miguel Farga, 1903. 419) convivían con "hermosas" y "felices ideas" (Díaz Muñoz, 1919, p. 119). Y, en el peor, "un verdadero arte de hacer salvajes", algo "diametralmente opuesto a un Tratado de Educación" (Ruiz Amado, 1911, p. 228). En último término, Emilio era el producto de un "vuelo imaginativo" (Escribano Hernández: 1925, p. 91), uno más de los "desvaríos del funesto enciclopedista" (Casas y Sánchez, 1910 , p. 95). No era un tratado pedagógico o didáctico; menos aún, la obra de un docente o maestro. El Emilio era una novela pedagógica, pura ficción imaginativa, imposible de llevar a la práctica, y Emilio un ser imaginario, repiten unos y otros con el objetivo de que los futuros maestros no se dejaran llevar por el estilo brillante, también reconocido, con que estaba escrito ${ }^{27}$.

Podía contener "observaciones prácticas y útiles" (Farga, 1903, p. 421), pero no era un ejemplo a seguir. Más bien, un contraejemplo. De ahí la insistencia con que en los manuales de orientación católico-conservadora se hacía referencia a sus múltiples errores y contradicciones, a su impractibilidad, a la condena de sus libros por la Iglesia católica, y a los anti-Emilios, en especial al del cardenal Gerdil. Más matizado sería el juicio de quienes, reconociendo algunos de los aspectos críticos antes mencionados, resaltaban, citando a Claparède y a Dewey, el gran número de ideas y principios aportados por Rousseau a la moderna ciencia de la educación (Gil Muñiz, Pertusa y Périz, 1930).

Y de muy diferente signo, de índole excepcional, sería el tratamiento recibido por Rousseau en el manual de Nicolás Escanilla: ninguna referencia condenatoria, relato de su vida exento de frases vejatorias o críticas, reconocimiento del carácter sistemático y completo de su obra, análisis detallado de sus aportaciones a la ciencia de la educación,

${ }^{26}$ Hay que tener en cuenta que, como ya se dijo, esta obra iba por la $12^{a}$ edición en los años 1960 . Las referencias al probable suicidio y a la frase citada se hallan también, por ejemplo, en RECUERO GARCÍA, Gutiérrez. Historia de la Pedagogía, op. cit., p. 116-117, SANTA MARÍA Y SÁENZ, Maria C. Historia de la educación y de la pedagogía. Badajoz, 1930, p. 171-172, y solo a la frase en ESCRIBANO HERNÁNDEZ, Godofredo. Historia general de la pedagogía y especial de la pedagogía española, op. cit., p. 243.

${ }^{27}$ MIGUEL FARGA, Luis. Compendio de historia de la pedagogía, op. cit., p. 421, GARCíA BARBARÍN, Eugenio. Historia de la pedagogía con un resumen de la Española, op. cit., p. 178, CASAS Y SÁNCHEZ, Manuel. Elementos de historia de la pedagogía, op. cit., p.96 y 97, y G. Escribano Hernández. Historia de la pedagogía, op. cit., p. 90. 
rechazo de la literatura sobre sus errores y de los anti - Emilios, y adscripción de Rousseau a la corriente de la educación natural dentro de la educación humana abstracta o general. Es otro Rousseau el que los estudiantes de Magisterio podían descubrir en sus páginas. En todo caso, Escanilla, por su pertenencia a la Federación Española de Trabajadores de la Enseñanza (Fete) de la Unión General de Trabajadores (UGT) desde 1918 y al Partido Comunista desde 1934, fue encausado, encarcelado y depurado, junto con su libro, tras la guerra civil.

\section{El predominio de la visión católico-conservadora durante el franquismo}

No tendría buena prensa Rousseau durante la dictadura franquista. A la tradicional condena sin paliativos de la Iglesia católica, se uniría el hecho de que José Antonio Primo de Rivera le calificara, en 1933, de "hombre nefasto" al comienzo del discurso fundacional de Falange Española. ${ }^{28}$ Ambas actitudes negativas hacia el ginebrino, reforzadas entre sí, determinarían la imagen de Rousseau transmitida por los manuales de Historia de la Pedagogía durante buena parte del franquismo. Una imagen que perduraría hasta la década de los 1970, aunque ya a fines de los 1960 apareciera algún libro de texto de signo más académico - científico y menos sectario.

"Rousseau no obraba conforme pensaba". Esta frase manuscrita de un o una estudiante de magisterio al comienzo del epígrafe dedicado a Rousseau, que figura en la edición manejada, de 1957, de la Historia de la Educación de Francisca Montilla, uno de los manuales más utilizados en las escuelas del magisterio durante los años 1940, 1950 y 1960 , condensa lo esencial de lo allí escrito y, posiblemente, de lo explicado en clase. ${ }^{29}$ En buena parte de los manuales, en especial en los de orientación más católicoconservadora, se atiende casi más, o más, a los avatares de la vida de Rousseau que a su obra. Las descalificaciones personales van junto con las pedagógico - educativas. Se repite su probable suicidio ${ }^{30}$ o se dice que "murió repentinamente, sin asistencia espiritual ni médica", (Montilla, 1957, p. 206) y se insiste de nuevo en su negativa a reconocer el pecado original y los dogmas de la Iglesia católica. A ello se añaden referencias a la condena en 1929 del naturalismo pedagógico en la encíclica Divini Illius Magistri de Pío XI.

Un juicio o valoración final negativa sobre su vida y su obra, se halla, en fin, tanto en las reediciones de la Historia de la educación de Ruiz Amado, como en obras publicadas en los años 1950 y 1960 y sucesivamente reimpresas, o en contestaciones a los temas de

${ }^{28}$ PRIMO DE RIVERA, José Antonio. Obras completas. Madrid, 1942, p. 17. Falange Española constituiría el núcleo básico, de índole fascista, del Movimiento Nacional, el partido único de la dictadura franquista. La frase completa sería ésta: "Cuando en marzo de 1762 un hombre nefasto, que se llamaba Juan Jacobo Rousseau, publicó El contrato social, dejó de ser la verdad política una entidad permanente". Es sintomático que desde 1936 - año de inicio de la guerra civil -, en que se publicaron las Cartas escogidas, hasta 1979, en que aparecieron los Escritos religiosos, no se publicara en España ninguna traducción de obras de Rousseau no traducidas con anterioridad.

${ }^{29} \mathrm{La}$ idea, expresada con toda seguridad de forma oral en clase, había sido ya mantenida por escrito años antes, como se ha dicho, al calificar a Rousseau como "la antítesis entre sus acciones y sus escritos" (Puigserver, 1918, p. 78).

30 SOLANA, Ezequiel. Curso completo de pedagogía. Cuarta parte. Historia de la pedagogía. Madrid: Escuela Española, 1944, p. 17. Ello se repetía en la edición de 1959, y en los temas de la reválida de magisterio y las contestaciones de las oposiciones de magisterio redactadas por dicha editorial en 1958 y 1960 respectivamente. 
la Reválida final o de oposiciones al Magisterio ${ }^{31}$. Otros autores contrastan, en su valoración final, la gravedad y número de los errores con la escasa relevancia y número de los aciertos ${ }^{32}$. Y otros, por último, equilibran, en relación con su obra, los errores y los aciertos $^{33}$. Como decía uno de ellos, Rousseau era "una cantera pedagógica de primer orden, que tiene, como todas las canteras, mucho mineral bueno, útil, de primera calidad, y mucha parte mala, inútil y de desperdicio" (Málaga García, 1947, p. 152).

En la segunda mitad de la década de los 60 aparecerían manuales menos sectarios y más académico - científicos que compartirían las aulas durante bastantes años con las reediciones de algunos ya mencionados como los de Montilla, Ruiz Amado, Edelvives y Gil Muñiz. Al fin y al cabo no podía desconocerse, aunque a más de uno le pareciera injusto, injustificado o paradójico que Rousseau fuera considerado "precursor de la Escuela Nueva", o "fundador de la pedagogía científica y padre de la educación nueva"34. Además, en los años 1960 se habían traducido y/o publicado en España e lberoamérica algunas historias de la educación o de la pedagogía en las que el tratamiento dado a Rousseau contrastaba claramente con el que era usual, por entonces, en los manuales españoles ${ }^{35}$. Conforme los historiadores de la educación españoles iban abriéndose hacia la producción exterior, era obvio que algo debía modificarse.

Así, en el manual de Celestino Sandín de 1967 se analizaba la obra de Rousseau, como portaestandarte del naturalismo, sin alusiones a su vida, sin descalificaciones personales o condenas y sin indicación de errores. Simplemente exponiendo su pensamiento e ideas a partir de su propia obra.

Todavía, sin embargo, quedarían restos o flecos de la versión católico-conservadora en manuales más modernizados publicados a finales de la década de los 1960 y en los primeros años de los 1970, y profusamente reeditados durante toda esta última década e incluso en los 1980. En el manual de Gutiérrez Zuluaga, por ejemplo, uno de los más utilizados en la enseñanza de esta disciplina y de orientación más modernizante, las referencias personales descalificadoras - por otra parte, muy moderadas en comparación con las efectuadas en manuales anteriores - quedaban reducidas a una nota inicial a pie

${ }^{31}$ Por ejemplo, en CAMPO PALACIO, V. Historia de la educación y de la pedagogía. Huesca, s. a., pero hacia 1945, p. 151, y Edelvives. Historia de la pedagogía. Zaragoza: Luis Vives, t. II, 1965, p. 27 - 39 (la editorial pertenecía a los Hermanos Maristas) y en las contestaciones a los temarios de la Reválida de 1958 y de las oposiciones de 1960 de la Editorial Escuela Española. En este último caso, las contestaciones fueron redactadas por un equipo en el que figuraba Víctor García Hoz, miembro del Opus Dei, catedrático de pedagogía y director del Instituto de Pedagogía San José de Calasanz del Consejo Superior de Investigaciones Científicas.

${ }^{32}$ Por ejemplo, VEGA ALONSO, R. Educación y su historia, s. I., s. a., pero hacia 1952, p. 159-160.

${ }^{33}$ Por ejemplo, en GIL MUÑIZ, Antonio. Estudios pedagógicos modernos. tomo tercero. elementos de historia de la pedagogía nociones de historia de la cultura. Málaga, 1960, p. 384-385, y LLOPIS, Jesús. Historia de la educación, op. cit., p. 180-181.

${ }^{34}$ FLORIANO CUMBREÑO, Antonio C. Los problemas educativos ante la historia: historia de la pedagogía, op. cit., t. II, p. 179, y CAMPO PALACIO, V. Historia de la educación y de la pedagogía, op. cit., p. 151. Hay que tener en cuenta que la casi totalidad de los manuales de los años 40, 50 e incluso 60, tenían por objetivo realzar la labor educativa de las órdenes y congregaciones religiosas y, en general, de la Iglesia católica, así como las obras e ideas de los pedagogos católicos. Para ello había que dejar a un lado, menospreciar o criticar la labor e ideas de quienes no encajaran en dicha línea u orientación.

${ }^{35}$ Por ejemplo, los de AGAZZI, Aldo. Historia de la filosofía y de la educación. Alcoy: Marfil, 1966, LARROYO, Francisco. Historia general de la pedagogía. México: Porrúa, 1944, ABBAGNANO, Nicola y VISALBERGHI, Aldo. Historia de la pedagogía. México: Fondo de Cultura Económica, 1964, LUZURIAGA, Lorenzo. Historia de la educación y de la pedagogía. Buenos Aires: Losada, 1967. 
de página, y las condenas, a una breve alusión al carácter "pernicioso" del concepto de educación negativa "desde el punto de vista de la virtud y de la religión" (Zuluaga, 1972, p. 294-298.) Y en el de Moreno, Poblador y del Río, publicado en 1971 y reimpreso todavía en 1986, se calificaban como "inmorales" algunos episodios de la vida de Rousseau, se aludía a las "nefastas consecuencias" de la idea de educación negativa, y se concluía el capítulo sobre el naturalismo pedagógico citando el párrafo de la encíclica Divini Illius Magistri en el que Pío XI condenaba "todo método de educación que se funde, en todo o en parte, sobre la negación u olvido del pecado original y de la gracia, y, por ello, sobre las fuerzas de la naturaleza humana" (Moreno; Poblador; D. del Río, 1974, p. 296, 298 y 303.)

\section{A modo de conclusión}

Los manuales de Historia de la Pedagogía o de la Educación con los que se ha formado, en este campo, el magisterio primario durante casi todo el siglo 20 - hasta el plan de 1991 en el que la sustitución de esta disciplina por la de Teoría e Instituciones Contemporáneas de la Educación dio origen a una nueva generación de libros de texto ofrecen, por lo general, una visión moralizante de Rousseau. Y, dentro de esa visión moralizante, ha predominado la católico-conservadora, en especial durante el franquismo. Rousseau figuraba en los manuales de la disciplina porque su influencia posterior en la génesis de lo que se llamaba la pedagogía moderna no podía ser desconocida. Pero figuraba como un contra - ejemplo, como un modelo a rechazar y condenar. $Y$, en el mejor de los casos, como autor de una obra contradictoria plagada de errores y aciertos.

Otras imágenes o visiones posibles de Rousseau, como la mantenida por la Institución Libre de Enseñanza, por quienes protagonizaron buena parte de la renovación pedagógica en la España del primer tercio del siglo 20 o, entre los autores de manuales de Historia de la Educación o Pedagogía, por Escanilla durante la II República, fueron cortadas de raíz por la guerra civil y el franquismo. Para encontrar visiones no sectarias ni condenatorias, sino simplemente expositivas o analíticas, habrá que esperar, y con carácter excepcional, a finales de los años 1960. Flecos y restos de esa concepción predominante pueden verse todavía en manuales de los años 1970 y 1980.

Restos sedimentados de esta corriente, en sus versiones más vulgares y simplistas, no en los trabajos académico-científicos, permanecen más o menos aletargados en el seno de la concepción católico-conservadora de la historia de la educación aflorando en los lugares y momentos más insospechados. Cuando el 26 de mayo de 1997, la flamante Ministra de Educación y Cultura, Esperanza Aguirre, pronunció en el club Siglo 21 una conferencia en la que, como es usual, criticaba la labor de sus predecesores y planteaba su programa de reformas, culpaba a Rousseau, haciéndonos recordar el discurso de 1933 del fundador de Falange Española, de ser el padre no solo de esa "importante corriente de la pedagogía [...], que es la que nos ha conducido a una desnaturalización, sistemática y letal, de la educación", sino también de "buena parte de las desgracias de nuestro sistema educativo moderno", lo cual, añadía, siguiendo lo dicho en la mayoría de los manuales analizados, no dejaba de ser "paradójico" en alguien que había abandonado a sus cinco hijos en "una institución de caridad" en el momento de nacer (Aguirre, 1988, p. 405). 
Que dichas frases fueran escritas por la ministra o por quien redactara el discurso, carece de importancia. Lo relevante es que Rousseau, para esa concepción católicoconservadora de la educación, sigue siendo, al mismo tiempo, la bestia negra de la educación moderna y el chivo expiatorio de todos sus supuestos males y desgracias.

\section{Referências}

ABENZA, A. Programa de historia de la pedagogía. Barcelona, 1932.

ABBAGNANO, N. y VISALBERGHI, A. Historia de la pedagogía. México: Fondo de Cultura Económica, 1964.

AGUIRRE, Esperanza. Educación y cultura: calidad y libertad. In: ALBERDI, Cristina et al. Democracia y alternancia: ciclo de conferencias 1996-1997. Madrid: Unión Editorial, 1988, p. 399-424.

ALONSO FERNÁNDEZ, L. Estudios elementales de pedagogía. Tomo segundo. Historia de la pedagogía. Madrid, 1946.

CAMPO PALACIO, V. Historia de la educación y de la pedagogía. Huesca, s. a.

CASAS Y SÁNCHEZ, Manuel. Elementos de historia de la pedagogía. Zaragoza: Talleres Tipográficos de P. Carra, 1910.

CONGRESO NACIONAL PEDAGÓGICO, Madrid, 1883 - intervención de Francisco Giner de los Ríos. In: BARNÉS, Domingo. Ensayos de pedagogía y filosofía. Madrid: La Lectura, s. a.

COSSíO, Manuel B. De su jornada: fragmentos. Madrid: Imprenta de Blass, 1929.

DE TUDELA, A. Programa - memorándum de historia de la pedagogía. Barcelona, $2^{\mathrm{a}}$ edición, 1919.

DE ZULUETA, Luis. El ideal en la educación: ensayos pedagógicos y otros textos. Madrid: Biblioteca Nueva, 2006.

DÍAZ MUÑOZ, Pedro. Historia de la pedagogía. Valladolid, 1919.

EDELVIVES. Historia de la Pedagogía. Barcelona: Luis Vives, s. a.

ESCRIBANO HERNÁNDEZ, Godofredo. Historia de la pedagogía. Madrid, 1910.

ESCRIBANO HERNÁNDEZ, Godofredo. Historia general de la pedagogía y especial de la pedagogía española. Madrid: Perlado Paez, 1925.

FIGUEROLA, Laureano. Guía lejislativa e inspectiva de instrucción primaria. Madrid: Europea de Hidalgo, 1844.

FLORIANO CUMBREÑO, Antonio C. Los problemas educativos ante la historia: historia de la pedagogía. Madrid: Magisterio Español, 1943.

GARCÍA BARBARÍN, Eugenio; RODRÍGUEZ GARCÍA, Gerardo. Historia de la pedagogía con un resumen de la Española. Madrid: Librería Hernando y Cía, 1907.

GINER DE LOS RÍOS, Francisco. Obras completas. XVI. Ensayos menores sobre educación y enseñanza. Tomo I, Madrid: Espasa-Calpe, 1927

GINER DE LOS RÍOS, Francisco. Obras completas. XII. Educación y enseñanza, Madrid: Espasa-Calpe, 1933.

GIL MUÑIZ, Antonio; PERTUSA Y PERIS, Vicente. Pedagogía moderna. Tomo III. Historia de la educación y de la pedagogía, Córdoba, 1930. 
GUTIÉRREZ ZULUAGA, Isabel. Historia de la educación. Madrid: Narcea, 1972.

LARROYO, Francisco. Historia general de la pedagogía. México: Porrúa, 1944.

LLOPIS, Jesús. Historia de la educación. Barcelona, 1969.

LUZURIAGA, Lorenzo. Historia de la educación y de la pedagogía. Buenos Aires: Losada, 1967.

MÁLAGA GARCÍA, Eduardo. Historia de la pedagogía. Burgos: Hijo de Santiago Rodríguez, 1947.

MIGUEL FARGA, Luis Compendio de historia de la pedagogía. Barcelona, 1903.

MONTESINO, Pablo. Curso de educación: métodos de enseñanza y pedagogía. Madrid: Ministerio de Educación y Ciencia, 1988.

MONTILLA, Francisca. Historia de la educación. Madrid: Escuela Española, 1957.

MORENO; POBLADOR; DEL RÍO: Historia de la educación. Madrid: Paraninfo, 1974.

PAROZ, Jules. Historia universal de la pedagogía. Gerona: Paciano Torres, 1889.

PRIMO DE RIVERA, José Antonio. Obras completas. Madrid, 1942.

PUIGSERVER, Maria. Apuntes de historia de la pedagogía. Castellón, 1918.

RABAZAS ROMERO, T. Los manuales de pedagogía y la formación del profesorado en las escuelas normales de España (1839-1901). Madrid: Uned, 2001.

RUIZ AMADO, Ramon. Historia de la educación y de la pedagogía. Barcelona, Gustavo Gili, 1911.

SANDÍN, Celestino. Historia de la educación. Alcoy: Marfil, 1967.

SANTA MARÍA Y SÁENZ, Maria C. Historia de la pedagogía y de la educación. Badajoz, 1930.

SPELL, Jefferson Rea. Rousseau in the spanish world before 1833. New York: Gordian Press, 1938.

SOLANA, Ezequiel. Curso completo de pedagogía: cuarta parte. Historia de la pedagogía. Madrid: Escuela Española, 1944.

SUREDA GARCÍA, Bernat. Introducción. En: MONTESINO, Pablo. Liberalismo y educación del pueblo. Madrid: Biblioteca Nueva, 2006.

UÑA, Juan. Discurso leído por el IImo. Sr. D. Juan Uña, rector de la Institución Libre de Enseñanza, en la inauguración del actual curso académico. Boletín de la Institución Libre de Enseñanza (Bile), n. 136, 1882, p. 221-223.

VEGA ALONSO, Rafael. Educación y su historia, s. I., s. a.

VILANOU TORRANO, Conrad; COLLELLDEMONT PUJADAS, Eulália. Presència de Rousseau a Catalunya: entre el mite y la realidad. Educació i Història, n. 19, 2012, p. 97152.

ZULUETA, Luis de. El ideal en la educación: ensayos pedagógicos y otros textos. Madrid: Biblioteca Nueva, 2006. 
ANTONIO VIÑAO FRAGO é catedrático de Teoria e História da Educação da Universidad de Murcia, Espanha. Seus campos de investigação prioritários são a história da alfabetização e da cultura escrita, da escolarização e do ensino secundário, assim como a história do currículo e a relação entre as culturas escolares e as reformas educativas.

Endereço: Universidad de Murcia - Facultad de Educación, 30080 - Murcia España.

E-mail: avinao@um.es.

Recebido em 29 de novembro de 2012.

Aceito em 30 de março de 2013. 